\title{
Decreased cardiac output: Diagnostic accuracy in heart transplant candidates
}

\author{
Lígia Neres Matos*1,2, Tereza Cristina Felippe Guimarães ${ }^{3}$, Viviani Christini da Silva Lima ${ }^{3}$, Ana Carla Dantas \\ Cavalcanti $^{4}$, Liana Amorim Correa Trotte ${ }^{2}$, Marcos Antônio Gomes Brandão ${ }^{2}$ \\ ${ }^{1}$ Pró Cardíaco Hospital, Rio de Janeiro, Brazil \\ ${ }^{2}$ Anna Nery School of Nursing, Federal University of Rio de Janeiro, Rio de Janeiro, Brazil \\ ${ }^{3}$ National Institute of Cardiology, Rio de Janeiro, Brazil \\ ${ }^{4}$ Aurora de Afonso Costa School of Nursing, Fluminense Federal University, Rio de Janeiro, Brazil
}

Received: April 24, 2019

DOI: $10.5430 /$ jnep.v9n11p19
Accepted: July 31, 2019

Online Published: August 9, 2019

\begin{abstract}
Objective: To identify the accuracy of non-invasive, defining characteristics for the nursing diagnosis of Decreased Cardiac Output among patients with heart failure.

Methods: Cross-sectional study. This study included 17 patients considered heart transplant candidates during the data collection period were selected (from May 2013 to May 2014). They were evaluated by the experts, and the cardiac output value was

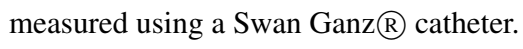

Results: Accuracy of the experts in diagnosing decreased cardiac output among patients with HF was high. Jugular vein stasis and electrocardiogram changes exhibited greater positive and negative predictive power for diagnosis.

Conclusions: It was concluded that jugular vein stasis and electrocardiogram changes were the defining characteristics that reached satisfactory efficiency values and were more relevant in producing correct classifications of the occurrence of decreased cardiac output by the moderate and high sensitivity and specificity of each defining characteristic.
\end{abstract}

Key Words: Heart failure, Cardiac output, Nursing diagnosis

\section{INTRODUCTION}

Heart Failure (HF), classically recognized as a clinical syndrome that expresses the inability of the heart to maintain the cardiac output necessary for adequate tissue perfusion, ${ }^{[1]}$ is "a complex clinical syndrome that can result from any structural or functional cardiac disorder that impairs the ability of the ventricle to fill with or eject blood". ${ }^{[2]}$

The prevalence of HF will increase by $46 \%$ from 2012 to 2030 according to projections, resulting in more than eight million people over 18 years of age diagnosed with $\mathrm{HF}^{[3]}$

Decreased cardiac output (DCO), defined as "inadequate blood pumped by the heart to meet the metabolic demands of the body", stands out among the relevant nursing diagnosis concepts expected in people with HF. The definition incorporates two main concepts: blood pumping and meeting metabolic demands. ${ }^{[4]}$

Considering the condition of heart failure, the consequences of decreasing cardiac output are frequent occurrences and of relevance for nurses dealing with patients in cardiovascular treatment units.

It should be emphasized that the measurement of cardiac output by invasive or non-invasive means contributes to the

*Correspondence: Lígia Neres Matos; Email: ligianeresmatos@yahoo.com.br; Address: Pró Cardíaco Hospital, Rio de Janeiro, Brazil. 
definition of diagnosis and therapeutic management. However, the nursing judgment will deal with the human response consequent to the functional and structural changes of the heart. Thus, it is opportune to consider in the diagnostic evaluation of nursing the occurrence of noninvasive clinical indicators (signs and symptoms) that guide the nurse in the decision to accept or discard the diagnosis.

The parameters of the pulmonary artery catheter provide accurate hemodynamic data and is considered the "gold standard" method for determination of filling pressures, postloading, cardiac index and continuous cardiac output. ${ }^{[5]}$ In a retrospective study evaluating $\mathrm{CD}$ prediction and decreased cardiac index, measured by pulmonary artery catheter, in patients with $\mathrm{HF}$, a higher predictive value of the following defining characteristics was found: third bulge and bradycardia in the category of noninvasive evidence. ${ }^{[6]}$

The gap in knowledge involves the accuracy of defining characteristics (DCs) in indicating the nursing diagnosis. Studies using the "gold standard" method for assessing cardiac output enable assessing the accuracy of DCs, thereby contributing to generate knowledge related to the nursing diagnosis of DCO and to the nurses' judgment by indicate the inferential validity of the noninvasive clinical indicators proposed by NANDA International Inc.

In a review study published in 2011, 79 DCO-related clinical indicators were identified, including 28 defining characteristics proposed by the taxonomy of NANDA International Inc. The following defining characteristics related to microcirculation assessment were notable: increased lactate levels and decreased venous oxygen saturation. ${ }^{[7]}$ Those that were associated with the evaluation of microcirculation were highlighted: increased lactate levels and decreased venous oxygen saturation.

Another study verified the most frequent defining characteristics of DCO: heart rate/frequency change, dyspnea, blood pressure lability, rales, oliguria/anuria, edema, cold skin, fatigue/weakness, peripheral pulse reduction and peripheral perfusion reduction. It presents the importance of the physical examination, the use of less invasive techniques and the need to review the defining characteristics. ${ }^{[8]}$ Knowing the accuracy of the defining characteristics of decreased cardiac output in patients with clinically compensated heart failure is relevant, and the results from this study may be used in clinical practice to treat patients with this hemodynamic profile. The aim was to identify the accuracy of non-invasive, defining characteristics for the nursing diagnosis of DCO among patients with heart failure who are heart transplant candidates.

\section{METHOD}

\subsection{Study sitting}

Department of Heart Failure and Heart Transplantation in a reference center in cardiology in the southeast of Brazil.

\subsection{Study design}

This is a cross-sectional study.

\subsection{Sample}

All patients considered heart transplant candidates during the data collection period were selected to avoid spectrum bias (from May 2013 to May 2014).

\subsection{Data collection}

After the ethical procedure, 17 patients older than 18 years with advanced HF, functional class III or IV of the New York Heart Association (NYHA), clinical treatment and maximum pharmacological and non-pharmacological treatments were included in the study. The patients should be clinically compensated, indicated for heart transplantation and taking no inotropic medication. Furthermore, two patients were excluded because they had clinically decompensated HF and were taking Dobutamine.

To collect data on DCs and nursing diagnosis decision, two nurse experts in patients' characteristics of the sample were selected. Those nurses were chosen based on Fehring criteria adapted to include nurses with clinical experience in nursing care in the area of interest for the diagnosis under study and care systematization. ${ }^{[9]}$

For each case, two experts independently evaluated patients using non-intrusive semiotic resources. In their evaluation, these experts should consider the presence or absence of defining characteristics of decreased cardiac output.

The following are the defining characteristics indicated by NANDA International Inc. evaluated non-invasively: electrocardiogram changes, bradycardia, tachycardia, palpitation, jugular vein stasis, edema, fatigue, crackles/rales, dyspnea, changes in skin color, cold and sticky skin, decreased peripheral pulses, prolonged peripheral capillary reperfusion, orthopnea, cough, paroxysmal nocturnal dyspnea, oliguria, restlessness and anxiety and B3 and B4 sounds. ${ }^{[4]}$

Given the subjective character of restlessness and anxiety, its consequent complexity and the limited time the experts had to establish a link during the data collection, oliguria, restlessness and anxiety were excluded from the list of DCs evaluated.

Cardiac index data were collected by direct, invasive monitoring using a flow-directed pulmonary artery catheter (Swan Ganz) and measuring the continuous cardiac output by a 


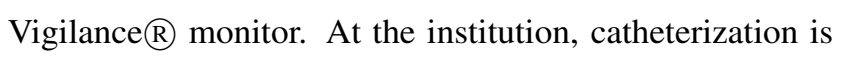
used to evaluate the pre-transplantation cardiac hemodynamic profile. Then, using the cardiac index (CI), cases with or without nursing diagnosis were related to the decreased (CI less than or equal to $2.51 / \mathrm{min} / \mathrm{m}^{2}$ ) or non-decreased (greater than $2.51 / \mathrm{min} / \mathrm{m}^{2}$ ) cardiac index. In all cases, data on invasive parameters were hidden from the experts.

\subsection{Statistical analysis}

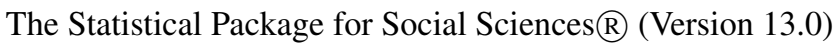
was used for data processing and analysis.

In addition to determining the presence or absence of decreased cardiac output, we sought to determine the specificity and sensitivity of each defining trait as we studied a population with a well defined and homogenous profile regarding the presence of heart failure and advanced stage of the disease. In this case, the use of the gold standard will contribute to validate the clinical diagnosis obtained through the physical examination by the experts. Sensitivity is understood as the probability of correctly identifying the presence of a clinical indicator (defining characteristic) in individuals with a nursing diagnosis; specificity is the probability of correctly identifying the absence of a clinical indicator (defining characteristic) in patients without the nursing diagnosis. The positive predictive value represents the probability of having the nursing diagnosis in patients with the defining characteristic. The negative predictive value indicates the probability of not having the diagnosis in patients without the defining characteristic. Accuracy (efficiency) represents the general measure that expresses the ability of a clinical indicator to accurately classify individuals with or without the target condition (DCO). The diagnostic odds ratio represents a measure of the increased chance of an individual being diagnosed when a given indicator (defining characteristic) is present.

\subsection{Ethics}

The confidentiality and anonymity procedures were followed, and data were only collected after the participants signed the informed consent form. The study was submitted to the Research Ethics Committee of National Institute of Cardiology - Rio de Janeiro/Brazil and approved under opinion number 738.907.

\section{Results}

Of the 17 patients included in the study, $14(82.3 \%)$ had a low cardiac index $\left(\mathrm{CI} \leq 2.5 \mathrm{l} / \mathrm{min} / \mathrm{m}^{2}\right)$ when hemodynamically evaluated using the pulmonary artery catheter.

The sample predominantly consisted of men with a mean age of 42 years $(\mathrm{SD} \pm 12.66)$ and functional class III. Table 1 outlines the distribution of the patients.

Table 1. Characterization of Participants According to Sex, Ethnicity and NYHA Functional

\begin{tabular}{|c|c|c|c|c|c|c|}
\hline \multirow{2}{*}{ Characteristics } & \multicolumn{2}{|c|}{$C I^{*} \leq 2.5 \mathrm{l} / \mathrm{min} / \mathrm{m}^{2}$} & \multicolumn{2}{|c|}{$\mathrm{CI}{ }^{*}>2.5 \mathrm{l} / \mathrm{min} / \mathrm{m}^{2}$} & \multicolumn{2}{|c|}{ Both } \\
\hline & $\mathbf{n}$ & $\%$ & $\mathbf{n}$ & $\%$ & $\mathbf{N}$ & $\%$ \\
\hline \multicolumn{7}{|l|}{ Sex } \\
\hline Male & 11 & 78.6 & 1 & 33.3 & 12 & 70.6 \\
\hline Female & 3 & 21.4 & 2 & 66.7 & 5 & 29.4 \\
\hline \multicolumn{7}{|l|}{ Ethnicity } \\
\hline White & 9 & 64.3 & 1 & 33.3 & 10 & 58.8 \\
\hline Brown & 4 & 28.6 & 2 & 66.7 & 6 & 35.3 \\
\hline Black & 1 & 7.1 & 0 & 0 & 1 & 5.9 \\
\hline \multicolumn{7}{|l|}{ Functional class } \\
\hline III & 13 & 92.9 & 3 & 100.0 & 16 & 94.1 \\
\hline IV & 1 & 7.1 & 0 & 0 & 1 & 5.9 \\
\hline
\end{tabular}

The most prevalent clinical and pathological features were ischemic $(35.3 \%)$ and idiopathic $(35.3 \%)$ cardiomyopathy. Coronary artery disease and arterial hypertension were diagnosed in $23.5 \%$ and $17.6 \%$ cases, respectively.

The experts reached a consensus about the absence and presence of diagnosis DCO in 12 of the 17 (70.6\%) patients evaluated. For each case, two experts independently evaluated patients using non-invasive semiotic resources. In their evaluation, these experts should consider the presence or absence of defining characteristics of decreased cardiac output.

Of these 12 patients, the pairs of experts who conducted the evaluations agreed on the presence of nursing diagnosis in 
10 cases $(83.3 \%)$. In 2 (two) cases the experts considered the absence of the diagnosis.

The overall efficacy of the expert classification of matching DCO cases (DCO:10 cases) and CI $\leq 2.5 \mathrm{l} / \mathrm{min} / \mathrm{m}^{2}$ (14 cases) was $71.4 \%$, which is considered acceptable to indicate the performance of the experts in relating the diagnostic construct to the objective measure of cardiac output.

The accuracy of the four nurses was verified in relation to the ability to relate the nursing diagnosis of DCO to the cardiac index. Two nurses achieved $100 \%$ accuracy, one nurse achieved $80 \%$ accuracy and another $28.5 \%$ accuracy. Considering the reduced sample size all the judgments were maintained for data analysis.
The mean number of defining characteristics was highest when the experts agreed to the existence of decreased cardiac output, regardless of the cardiac index measurement. In the 10 matching DCO cases, the mean number of defining characteristics/patient assessed was 4.50 defining characteristics/patient in the group with diagnosis and 0.75 defining characteristics/patient in the group without diagnosis. The mean was 2.6 defining characteristics/patient in the five cases without agreement between experts (uncertainty).

Table 2 outlines the measures of the diagnostic test calculated for each defining characteristic that were identified in the patients.

Table 2. Measures of accuracy of the defining characteristics of decreased cardiac output

\begin{tabular}{|c|c|c|c|c|c|c|c|}
\hline Defining characteristics & N (\%) & Sens & Specif. & PPV & NPV & EF (\%) & OR \\
\hline \multicolumn{8}{|l|}{ 1. Altered heart rate/frequency } \\
\hline ECG changes & $7(41.2)$ & 50.0 & 100 & 100 & 50.0 & 66.6 & 9.0 \\
\hline Bradycardia & $2(11.8)$ & 37.5 & 100 & 100 & 33.3 & 58.3 & 5.7 \\
\hline Tachycardia & $1(5.9)$ & 12.5 & 100 & 100 & 36.6 & 41.6 & 1.8 \\
\hline Palpitations & 0 & & - & - & - & - & - \\
\hline \multicolumn{8}{|l|}{ 2. Altered preload } \\
\hline Jugular vein stasis & $7(41.2)$ & 75.0 & 75.0 & 85.7 & 60.0 & 75.0 & 6.1 \\
\hline Edema & $2(11.8)$ & 25.0 & 100 & 100 & 40.0 & 50.0 & 3.5 \\
\hline Fatigue & $3(17.6)$ & 12.5 & 100 & 100 & 36.6 & 41.6 & 1.8 \\
\hline Crackles/rales & $2(11.7)$ & 25.0 & 100 & 100 & 40.0 & 50.0 & 3.5 \\
\hline \multicolumn{8}{|l|}{ 3. Altered post-load } \\
\hline Dyspnea & 0 & - & - & - & - & - & - \\
\hline Change in skin color & $3(17.6)$ & 37.5 & 100 & 100 & 33.3 & 58.3 & 5.7 \\
\hline Cold and sticky skin & 0 & - & - & - & - & - & - \\
\hline Decreased peripheral pulses & $3(17.6)$ & 25.0 & 100 & 100 & 40.0 & 50.0 & 3.5 \\
\hline Prolonged peripheral capillary reperfusion & $2(11.8)$ & 25.0 & 100 & 100 & 40.0 & 50.0 & 3.5 \\
\hline \multicolumn{8}{|l|}{ 4. Altered contractility } \\
\hline Crepitations & $3(17.6)$ & 37.5 & 100 & 100 & 33.3 & 58.3 & 7.0 \\
\hline Paroxysmal nocturnal dyspnea & 0 & - & - & - & - & - & - \\
\hline Orthopnea & $2(11.8)$ & 12.5 & 100 & 100 & 36.6 & 41.6 & 1.8 \\
\hline B3 Sounds & $7(41.2)$ & 37.5 & 50.0 & 62 & 33.3 & 41.6 & 0.6 \\
\hline B4 Sounds & 0 & - & - & - & - & - & - \\
\hline Cough & $3(11.8)$ & 25.0 & 100 & 100 & 40.0 & 50.0 & 3.5 \\
\hline
\end{tabular}

Note. Sens: sensitivity; Specif.: specificity; PPV: positive predictive value; NPV: negative predictive value; EF:efficiency (accuracy); OR: Diagnostic odds ratio.

\section{Discussion}

In contrast to the findings of this study in which the sample of patients with HF predominantly consisted of men with a mean age of 42 years, the mean age of patients with HF in a study conducted in Brazil was $64 \pm 16$ years. In addition,
$73 \%$ were older than 75 years, and $60 \%$ were women. ${ }^{[10]}$ In conditions similar to those of the present study in which were to identify the prevalence of DC of DCO in patients with cardiac insufficiency under evaluation for heart transplantation $71 \%$ of the patients were female and average age 
was 47 years. $^{[6]}$

Regarding the interpretation of the accuracy of the tests, the high prevalence of DCO in the opinion of the experts is notable but nevertheless lower than the occurrence of decreased cardiac index. The difference observed is likely explained by differences in the construct evaluated.

With respect to the construct, the decreased cardiac output is a clinical judgment on a human response expressed in clinical indicators used as beacon for the nurse's decision. Conversely, the cardiac index is an objective variable of a physical phenomenon calculated from measurements of other physical properties. Therefore, a subjective language construct is compared with an objective measure directly referenced in the empirical phenomenon.

Regarding the accuracy measures, the high values for specificities in several CDs point to a greater probability of not being able to determine the defining characteristics in patients without DCO. In combination with high positive predictive values (PPV), this finding would suggest that identifying the defining characteristics could be a criterion to increase the nurses' confidence in considering the presence of the diagnosis when assessing the clinical indicator. Because the patients are clinically compensated, many of these findings would presumably be assessed only when a considerable change in cardiac output would generate verifiable modifications by noninvasive analysis.

Conversely, the low negative predictive values (NPV) observed in several DCs prevent using their absence as a sufficient criterion to discard DCO. Such findings suggest that the absence of evidence (DC) alone would not have sufficient predictive power to discard the diagnosis in such a specific population and with a high degree of cardiac involvement. The strength of the experts' experience may have placed pressure on the predictive model of nursing diagnosis based on defining characteristics.

The tendency to rely on the presence of a characteristic to give the diagnosis and not rely on its absence to exclude the diagnosis may be an explanatory factor for the only moderate values of accuracy (efficiency) of most CD.

In general, the likely tendency to consider decreased cardiac output highly prevalent was not overestimated because decreased cardiac index was assessed in $82.3 \%$ cases.

The nursing academic community in Brazil has been developing studies that contribute to the refinement by nurses in the identification of the diagnosis of decreased cardiac output in the population with HF. ${ }^{[6]}$ In a study of verification of accuracy in the determination of nursing diagnoses fatigue,

Published by Sciedu Press intolerance to activity and DCO in patients with hospitalized $\mathrm{HF}$, conducted with experts, the authors concluded that despite the similarity between nursing diagnoses fatigue, DCO, and intolerance to activity, which have common characteristics and the three diagnoses could be identified between the cases applied and can be guaranteed statistically effectiveness. ${ }^{[11]}$

The lack of confidence in discarding the diagnosis due to the absence of given evidence could be overcome by a deeper search for other clinical indicators (DCs). Because the DCO spectrum is not that exacerbated in those "compensated" patients, monitoring the worsening of clinical symptoms could identify the onset of new defining characteristics. Thus, by combining two or more DCs, the nurse would gain confidence in diagnosing the DCO and would noninvasively detect early symptoms of decompensation.

More specifically, considering the diagnostic odds ratios that would associate the DC with the diagnosis in a summary measure, the following defining characteristics are more likely associated with diagnosis: stasis of jugular veins, changes in ECG, change in skin color and crackling. These characteristics were also the four most efficient DCs in classifying the true positives and true negatives.

In conditions similar to those of the study was possible to verify the most frequent $\mathrm{DC}$ when the $\mathrm{CI}$ less or equal to $2.5 \mathrm{l} / \mathrm{min} / \mathrm{m}^{2}$ was present: tachycardia $(100 \%)$, bradycardia $(75 \%)$ and increased and decreased central venous pressure (both with $66.7 \%$ ), which corroborates our findings regarding the altered heart rate/frequency and altered preload. ${ }^{[6]}$

The clinical validation of the diagnosis of decreased cardiac output in different conditions nurses address in their practice is still far from completion. To make the validity of the diagnosis more sustainable, when possible, it would be desirable to perform standardized tests that would serve as a basis for the assessment of the accuracy of the diagnostic judgment. In the context of the diagnosis of DCO, the gold standard would represent the value of cardiac output measured by appropriate equipment (invasive or non-invasive).

In the first diagnostic validation studies, ${ }^{[12,13]}$ DCO was more commonly identified in patients with acute myocardial infarction and coronary heart disease, and greater than 180 defining characteristics were related to the presence of decreased cardiac output.

Corroborating the above findings, in a study conducted with 29 patients with heart failure, albeit decompensated, and using the Fehring model, the following characteristics were considered increased $(\geq 0.80)$ : fatigue, dyspnea, edema, orthopnea, paroxysmal nocturnal dyspnea and increased central 
venous pressure. ${ }^{[14]}$

\subsection{Limitation}

One of the limitations of study is the small sample of the population. Consideration should be given to the specificity of the population profile studied.

\subsection{Clinical implications}

Despite these limitations, results from this study have important clinical implications. In the present study, the patients had a clinically compensated profile. Patients were undergoing drug therapy optimized for advanced-stage heart failure and followed in a multidisciplinary outpatient clinic to ensure weight control, water restriction and drug use guidance. This feature may have minimized the exacerbation of some of the study symptoms that were not identified, such as dyspnea and cold and sticky skin, which are likely more expected in clinical decompensation.

Finally, because the diagnosis of DCO has more than three dozen DCs proposed by the taxonomy of NANDA International Inc. (2015), clinicians should sensibly consider that such characteristics are not random. Therefore, the higher the number of those indicators, the closer to the nursing diagnosis construct they will likely be. The likely result of this reasoning is gaining confidence when the patient exhibits more than one defining characteristic.

\section{Conclusion}

In conclusion, the accuracy of the experts in diagnosing decreased cardiac output among patients with HF was high.
Jugular vein stasis and altered ECG were the defining characteristics that reached satisfactory efficiency values and were more relevant in producing correct classifications of the occurrence of decreased cardiac output by the moderate and high sensitivity and specificity of each defining characteristic.

In this study, the presence of four or more defining characteristics was strongly related to the presence of a decreased cardiac output and was corroborated by the low cardiac index.

This finding leads us to conclude that even the patient with low cardiac index, adaptation mechanisms, promoted by pharmacological treatment and non- pharmacological treatment, are fundamental for these patients to remain in functional class III (NYHA).

Finally, the defining characteristics of DCO in patients with clinically compensated heart failure who are heart transplant candidates, directs the nurses' pointing out the no-invasive clinical indicators proposed by NANDA International Inc.

Therefore, it is concluded that the validation of the defining characteristics for the nursing diagnosis of DCO in patients with clinically compensated heart failure is relevant, and the results of this study may be used in clinical practice in the care of patients with this hemodynamic profile.

\section{CONFlicts of InTEREST Disclosure}

The authors have no conflict of interest to declare.

\section{REFERENCES}

[1] Hess OM, Carolle JD. Clinical assessment of heart failure. In P. Libby, R. O. Bonow, D. L. Mann, \& D. P. Zipes (Eds.), Braunwald's heart disease: A textbook of cardiovascular medicine. 8th ed.. Philadelphia: Elsevier; 2008.

[2] Writing Committee Members, Yancy CW, Jessup M, Bozkurt B, et al. American College of Cardiology Foundation/American Heart Association Task Force on Practice Guidelines. 2013 ACCF/AHA guideline for the management of heart failure: A report of the American college of cardiology foundation/American heart association task force on practice guidelines. Circulation. 2013; 128(16): e240-e327.

[3] Writing Group Members, Mozaffarian D, Benjamin EJ, Stroke Statistics Subcommittee. Heart disease and stroke statistics-2016 update: A report from the American heart association. Circulation. 2016; 133(4): e38-e36

[4] NANDA International Inc. Diagnóstico de enfermagem da NANDA internacional: Definições e classificações. Débitoc cardíaco diminuído (2015-2017) [Nursing diagnoses: Definitions \& classifcations 2015-2017. Porto Alegre: Artmed. 2015.

[5] Flato UAP, Campos AL, Trindade MR, et al. Intensive care bedside echocardiography: true or a distant dream? Rev Bras Ter Intensiva. 2009; 21(4): 437-444.

[6] Matos LN, Guimarães TCF, Brandão MAG, et al. Prevalence of nursing diagnosis of decreased cardiac output and the predictive value of defining characteristics in patients under evaluation for heart transplant. Revista Latino-Americana de Enfermagem. 2012; 20(2): 307-315. PMid:22699731 https ://doi .org/10.1590/S0104-1 1692012000200013

[7] Brandao SM, Altino DM, e Silva Rde CG, et al. Defining characteristics of decreased cardiac output: A literature review. International Journal of Nursing Terminologies and Classifications. 2011; 22(2): 92-102. PMid:21521458 https : //doi.org/10.1111/j.1744-6 18X.2010.01174.X

[8] Souza V, Sandra SZ, Barros ALBL. Decreased cardiac output: a systematic review of the defining characteristics. Act Paul Enferm. 2011; 24(1): 114-9.

[9] Martins QCS, Aliti GB, Linhares JC, et al. Excess fluid volume: Clinical validation in patients with decompensated heart failure. Revista Latino-Americana de Enfermagem. 2011; 19(3): 540-547. https ://doi.org/10.1590/S0104-11692011000300013 
[10] de Albuquerque DC, de Souza Neto JD, Bacal F, et al. I registro brasileiro de insuficiência cardíaca - aspectos clínicos, qualidade assistencial e desfechos Hospitalares [I brazilian registry of heart failure - clinical aspects, care quality and hospitalization outcomes]. Arquivos Brasileiros de Cardiologia. 2015; 104(6): 433-442.

[11] Pereira JMV, Cavalcanti ACD, Lopes MVO, et al. Accuracy in inference of nursing diagnoses in heart failure patients. Rev Bras Enferm. 2015; 68(3): 603-9.

[12] Dahon J. A descriptive study: Defining characteristics of the nursing diagnosis cardiac output, alterations in: Decreased. Image: the
Journal of Nursing Scholarship. 1985; 17(4): 113-117. https: //doi.org/10.1111/j.1547-5069.1985.tb01638.x

[13] Dougherty CM. Decreased cardiac output: Validation of a nursing diagnosis. Dimensions of Critical Care Nursing. 1986; 5(3): 183-188. https://doi.org/10.1097/00003465-198605000-00009

[14] Martins QC, Aliti G, Rabelo ER. Decreased cardiac output: Clinical validation in patients with decompensated heart failure. International Journal of Nursing Terminologies and Classifications. 2010; 21(4): 156-165. PMid:20977620 https ://doi.org/10.1111/j. 1744-618X.2010.01161.x 